\title{
Advanced cognitive networked radar surveillance
}

\author{
Mohammed Jahangir, Chris J Baker, \\ Michail Antoniou, \\ EESE Dept, University of Birmingham \\ Birmingham, UK \\ m.jahangir@bham.ac.uk, \\ c.j.baker.1@,bham.ac.uk, \\ m.antoniou@bham.ac.uk
}

\author{
Benjamin Griffin, Alessio Balleri \\ Centre for EWIC, Cranfield University, \\ Defence Academy of the UK \\ Shrivenham \\ b.griffin@cranfield.ac.uk, \\ a.balleri@,cranfield.ac.uk
}

\author{
David Money, Stephen Harman \\ Thales \& Aveillant \\ Cambridge, UK \\ David.money@uk.thalesgroup.com , \\ stephen.harman@aveillant.com
}

\begin{abstract}
The concept of a traditional monostatic radar with co-located transmit and receive antennas naturally imposes performance limits that can adversely impact applications. Using a multiplicity of transmit and receive antennas and exploiting spatial diversity provides additional degrees of design freedom that can help overcome such limitations. Further, when coupled with cognitive signal processing, such advanced systems offer significant improvement in performance over their monostatic counterparts. This will also likely lead to new applications for radar sensing. In this paper we explore the fundamentals of multistatic network radar highlighting both potential and constraints whilst identifying future research needs and applications. Initial experimental results are presented for a 2-node networked staring radar.
\end{abstract}

Keywords-Networks, Multistatic, Distributed, Cognitive, Intelligent, Radar

\section{INTRODUCTION}

Traditional monostatic radar, with transmission and reception via a single aperture has, in general, been the default design for almost as long as radar sensing has been around. It has the advantages of minimising equipment quantity and size and many radar characteristics are simplified. For example, ranging and detection performance are described using a circular geometry with the radar at the centre of the circle. Nevertheless, the monostatic configuration imposes limits that, potentially, can be overcome with a network of radar sensors.

At the simplest level, a network may be thought of as the combination of outputs from multiple monostatic radars. There are numerous examples where this has been implemented, driven by particular system level performance gains as well as extended and contiguous coverage. The wellknown US NEXRAD network of weather radars and its European counterpart OPERA are good examples [1, 2]. NEXRAD consists of around 160 radar sites, covering continental USA and provides continuous monitoring of weather phenomena in both space and time. OPERA has over 200 radar stations spread across more than 20 countries. Both supply a wide range of weather products although they achieve this in significantly different ways.

These networks of radars designed for detecting and tracking weather phenomena have enabled further exploitation in other applications areas. For example, aero ecology is a fast-growing field that examines the aerial movement of organisms and their interactions with the aerial environment, birds, bats and insect being most commonly observed [3]. Processing based networks, such as the European Network for the Radar surveillance of Animal Movement (ENRAM) have been firmly established. These focus on the specific processing requirements of networked weather radar data to both enhance quality and coverage of observations.

Ocean monitoring, driven by the need to feed climate models as well as providing real-time assessments has led to rapid growth in a networked approach. Indeed, the goal is to have complete and comprehensive global coverage [4].

In fact, radar networks are rapidly becoming all pervasive finding applications in remote sensing, provision of perception for autonomous transportation, health monitoring, security and military applications and many more. An excellent and up to date overview is provided in [5]. Here we exclusively use the definition of the network to be a collection of multistatic nodes linked coherently. This is different from multi-site networks that operate in-coherently and can offer benefits but are outside the scope of this paper.

Advances is radar hardware are leading to equipment that can be lower cost and flexible in parameter selection. As a consequence, fully coherent networks in which transmitters and receivers are distributed in space, as opposed to a series of connected monostatic radars have started to become of interest. Examples include the Netrad [6] and Nextrad [7] systems developed in the UK. These offer opportunity both to investigate hardware design as well as providing a source of gathering networked data observations of targets and clutter, both in scarce supply. The CREW radar system [8] was developed as a networked radar capable of cognitive processing both in terms of hardware adaptation and processing adaptation. This is the first example of the specific design of a cognitive networked radar system.

The advantages of coherent networks are in improved sensitivity, enhanced target detection, reduced dynamic range requirements and richer forms of target and clutter echoic information.

Overall, radar networks offer potential performance advantage through additional degrees of freedom afforded by using multiple transmitters and receivers separated in space. In recent years, the advantages of using a multiplicity of radars to form a spatially distributed sensor have led to a sustained increase in research and development. In parallel, there has been an upsurge in advanced processing of radar echoes exploiting feedback, memory, and prior information, all coming under the banner cognitive. The combination of cognitive signal processing and the distribution of sensor elements over space offers yet further potential benefits. Here we examine aspects of coherent, cognitive radar networks concentrating on performance potential and real-world implementation. Specifically, we introduce the relationship between transmitter and receiver location and achievable levels of performance. We then investigate the real-world design and implementation of a network using a staring geometry as a means of establishing hardware performance, 
particularly the levels of achievable coherency, as a function of design approach.

The remainder of this paper is organised as follows. Section II investigates the optimal pairing of receiver arrays in a network for minimising the position and velocity estimation error for a target. Section III describes the hardware set for initial field trials using a two node multistatic network of staring radar and presents result from small light aircraft used as the reference target. Finally, the conclusions are given in Section IV.

\section{GEOMETRY AND PERFORMANCE}

A multistatic radar network's ability to estimate a target position and velocity depends on its geometry, relative to the target. Previously, a study into finding the geometry that minimises the position and velocity estimation error has been completed [18]. Here, building on the previous work, a method for selecting the optimal combinations of measurements from a network is presented. This section contains three subsections. Firstly, the Cramér Rao Lower Bound (CRLB) for estimating a targets position and velocity from a network is presented. Secondly, the receiver selection algorithm is introduced and applied to a given network geometry. Finally, the algorithm is applied to a simulated target trajectory.

\section{A. Target Position and Velocity Estimation Error}

A two-step method for estimating a 2D target position and velocity from a network is summarised [18]. Firstly, each receiver independently estimates the target relative range and Doppler shift. Secondly, the target range and Doppler MLE estimates are centrally combined to estimate, the vector parameter $(\boldsymbol{\theta})$ that describes the deterministic unknown target position and velocity, using the asymptotic properties of MLE estimators.

$$
\boldsymbol{\theta}=\left(x, y, v_{x}, v_{y}\right)
$$

Consider a radar network consisting of a single transmitter and $\mathrm{N}$ omnidirectional receivers. The received signal at each receiver will be time delayed and Doppler shifted depending on the geometry of the radar network and the relative position and velocity of the target. The received signal after down conversion may be expressed as:

$$
s_{n}(t)=\sqrt{E_{n}} u\left(t-\tau_{n}(\boldsymbol{\theta})\right) e^{2 \pi i f_{n}(\boldsymbol{\theta}) t}+w_{n}(t)
$$

where $\tau_{n}(\boldsymbol{\theta}), f_{n}(\boldsymbol{\theta})$ and $E_{n}$ represent the time delay, Doppler shift and the energy received at the nth receiver, the complex envelope $u(t)$ is assumed to be unitary. The signal noise $w_{n}(t)$ is modelled as complex zero-mean Gaussian random process. The estimation performance of the time delay and Doppler shift from (2) is well known and depends on the signal to noise ratio (SNR) and the ambiguity function of the complex envelope [9]. The MLE estimator of range and Doppler can asymptotically be modelled as Gaussian random variables:

$$
\begin{aligned}
& \hat{r}_{n}(\boldsymbol{\theta})=r_{n}(\boldsymbol{\theta})+e_{r_{n}} \\
& \hat{f}_{n}(\boldsymbol{\theta})=f_{n}(\boldsymbol{\theta})+e_{f_{n}}
\end{aligned} \quad \Sigma_{n}=\frac{1}{\mathrm{SNR}_{n}}\left(\begin{array}{cc}
\sigma_{r}^{2} & \sigma_{r f} \\
\sigma_{f r} & \sigma_{f}^{2}
\end{array}\right)
$$

where $\hat{r}_{n}$ and $\hat{f}_{n}$ are the MLE estimates of the target range and Doppler shift at the nth receiver $(\hat{r}=c \hat{\tau}$ where $c$ is the speed of light). These measurements are dependent on the target parameter vector and the geometry of the radar network. The range and Doppler shift measurements from the

$$
\boldsymbol{z}=\boldsymbol{\mu}(\boldsymbol{\theta})+\boldsymbol{e}=\left(\begin{array}{c}
r_{1}(\boldsymbol{\theta}) \\
f_{1}(\boldsymbol{\theta}) \\
\vdots \\
r_{N}(\boldsymbol{\theta}) \\
f_{N}(\boldsymbol{\theta})
\end{array}\right)+\left(\begin{array}{c}
e_{r_{1}} \\
e_{f_{1}} \\
\vdots \\
e_{r_{N}} \\
e_{f_{N}}
\end{array}\right)
$$

radar network are combined into a single vector (4).

The vector $\boldsymbol{z}$ is a Gaussian random vector with mean value $\boldsymbol{\mu}(\boldsymbol{\theta})$ and covariance matrix $\boldsymbol{\Sigma}$. As the measurement noise signals in (2) from each receiver are independent, the measurement errors are also independent and the covariance matrix of $\mathbf{z}$ is a block diagonal matrix (5).

$$
\boldsymbol{\Sigma}=\operatorname{blkdiag}\left(\Sigma_{1}, \Sigma_{2}, \ldots, \Sigma_{\mathrm{N}}\right)
$$

Each diagonal block contains the range and Doppler shift covariance matrix from each individual receiver in the network. As $\boldsymbol{\Sigma}$ is a block diagonal matrix, the Fisher Information Matrix (FIM) of the estimation of the target parameter vector $(\boldsymbol{\theta})$ from (3) may be written as: [10]

$$
\begin{aligned}
& {[I(\boldsymbol{\theta})]_{i j}=\sum_{n=1}^{N}\left[\frac{\partial \boldsymbol{\mu}_{n}(\boldsymbol{\theta})}{\partial \theta_{i}}\right]^{\mathrm{T}} \Sigma_{n}^{-1}\left[\frac{\partial \boldsymbol{\mu}_{n}(\boldsymbol{\theta})}{\partial \theta_{j}}\right]} \\
& {\left[\frac{\partial \boldsymbol{\mu}_{n}(\boldsymbol{\theta})}{\partial \theta_{i}}\right]=\left[\frac{\frac{\partial r_{n}(\boldsymbol{\theta})}{\partial \theta_{i}}}{\frac{\partial f_{n}(\boldsymbol{\theta})}{\partial \theta_{i}}}\right]}
\end{aligned}
$$

The CRLB is the inverse of the FIM (6) and defines a bound on the covariance of an unbiased estimator of the target parameter vector using the networks range and Doppler measurements. The CRLB depends on the network geometry and the target position and velocity of the target.

\section{B. Optimal Receiver Pair Selection}

Consider a scenario in which the measurements of only a subset of the network's receivers can be used to estimate the target position and velocity. The optimal subset of receivers are the receivers which result in the smallest possible estimation error of the target parameter vector. The trace of the position and velocity CRLB can be used as a cost function to compare the estimation performance of different subsets of receivers for a given target position and velocity.

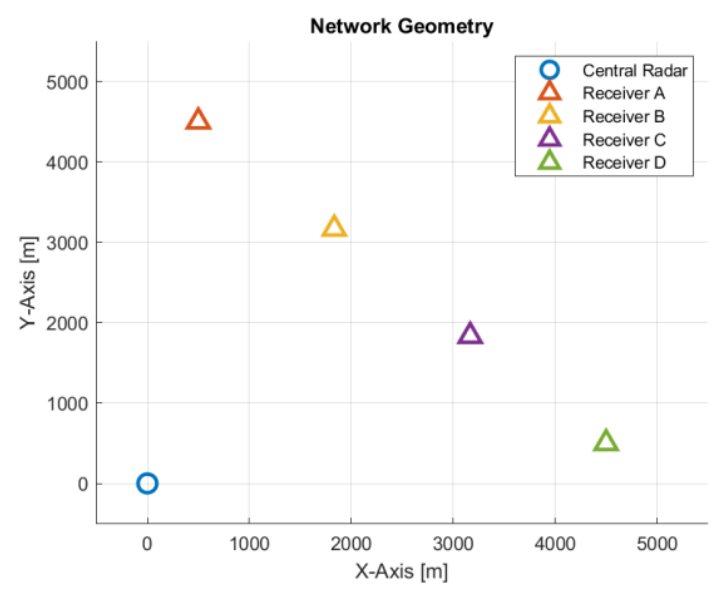

Fig. 1 Network Geometry

Fig. 1 illustrates a radar network consisting of a central radar and four smaller omnidirectional bistatic receivers. The central radar is based on the performance of the Aveillant Gamekeeper radar and each bistatic receiver is based on the performance of a single Aveillant channel [14]. Here, we consider how to select, for a given target position and 
velocity, which pair of the bistatic receivers to use in combination with the central radar.

The receiver selection algorithm evaluates, for a given target position and velocity, the trace of the position and velocity CRLB using the measurements from each pair of bistatic receivers in combination with the measurements from the central radar and selects the pair of bistatic receivers that minimises the resulting trace. For the radar network shown in Fig.1, there are six possible combinations of receiver pairs.

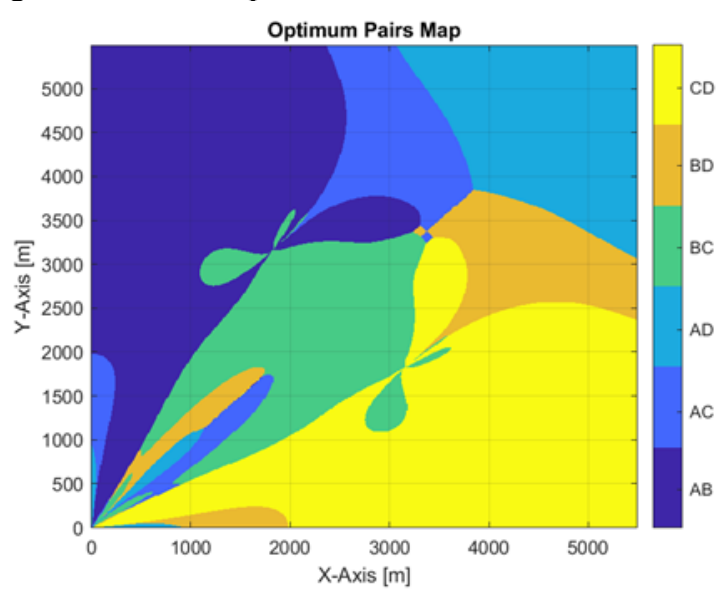

Fig. 2 Optimum receiver pair map

Fig. 2 shows a colour coded slice of the optimal pair map for the network illustrated in Fig. 1. The slice corresponds to drone like a target that has a velocity of $-10 \mathrm{~ms}^{-1}$ in both the $x$ direction and $y$ direction. Each colour corresponds to a different pair of receivers. Note, that the map is symmetrical as the target has the same velocity in both the $x$ and $y$ direction.

\section{Simulated Trajectory.}

This sections applys the selection algorthm to a simulated trajectory. For each point in the trajectory the optimal receiver pairings is calculated based on the targets position and velocity relative to the network.

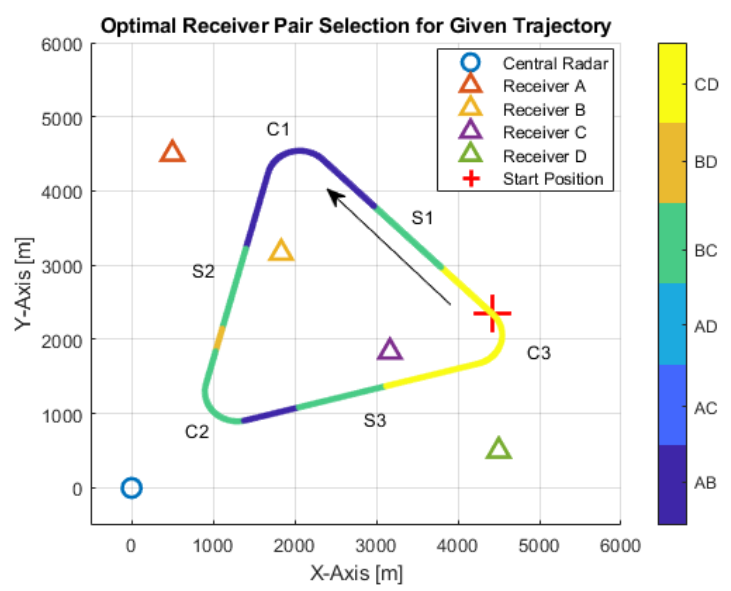

Fig. 3 Optimal receiver pair selection for a simulated trajectory

Fig. 3 shows a simulated trajectory of a drone like target, colour coded according to the optimal receiver pairings. The target moves in an anticlockwise direction along the trajectory. The targets speed along the three straight sections (labelled S1 to S3) is held constant at $10 \mathrm{~ms}^{-1}, 30 \mathrm{~ms}^{-1}$ and $5 \mathrm{~ms}^{-1}$ respectively. The target speed is interpolated on the corners (labelled $\mathrm{C} 1$ to $\mathrm{C} 3$ ) to transition from the speed of the previous section to the next section.

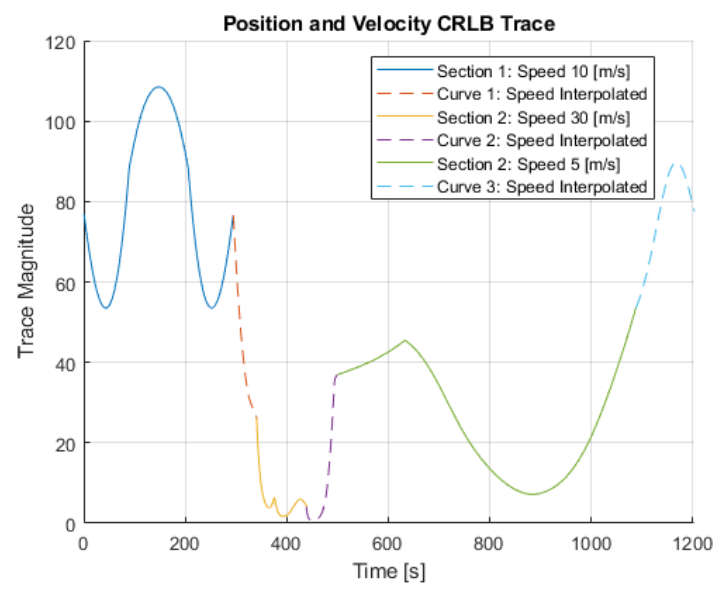

Fig. 4 Trace of the position and velocity CRLB over time

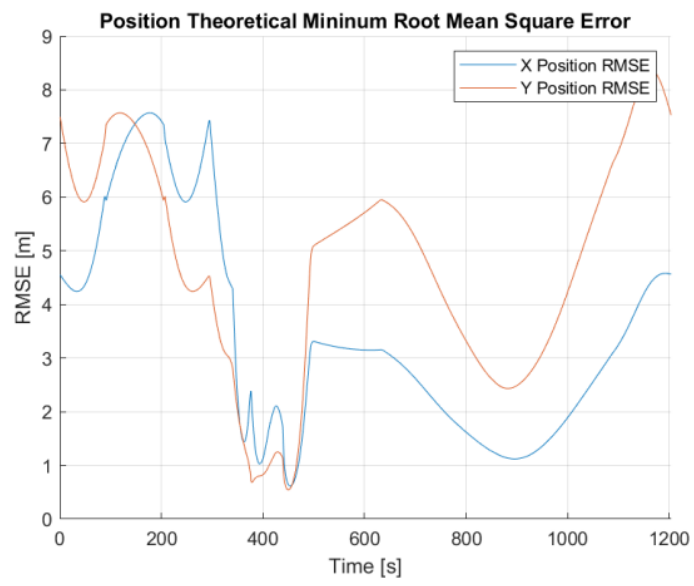

Fig. 5 Position theoretical minimum RMSE

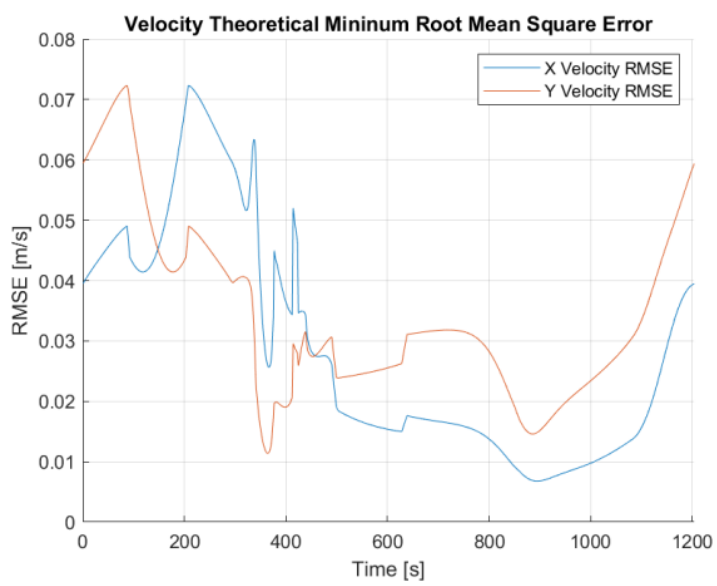

Fig. 6 Velocity theoretical minimum RMSE

Fig. 4 shows the trace of position and velocity CRLB for the simulated trajectory. The solid lines correspond to times when the targets speed is constant (S1-3), and dotted lines correspond to corners (C1-3). Fig. 5 and 6 show the theoretical root mean square error (RMSE) for the position and velocity components of the CRLB trace. The minimum position RMSE ranges from $8 \mathrm{~m}$ to $1 \mathrm{~m}$, and the minimum velocity RMSE ranges from $0.01 \mathrm{~ms}^{-1}$ to $0.07 \mathrm{~ms}^{-1}$. In both plots, there are fluctuations between $250 \mathrm{~s}$ and $500 \mathrm{~s}$; this period corresponds to times when the targets speed is highest, and therefore, its position and velocity are more rapidly changing. 


\section{Summary}

This section has explored a method for using the CRLB of a position and velocity estimator to determine the optimal pairings of receivers. This work can be applied to cooperative scenarios where the incoming trajectory is known to a greater degree of confidence (landing at airports) where the measurements from additional bistatic receivers are selected to improve estimation performance along specific parts of the trajectory. Currently, only range and Doppler measurements have been exploited; however, the model can be extended to include additional measurements, such as angle of arrival (AOA). The model can also be expanded to include real-time knowledge of the environment and the effects of interference and multipath propagation on the received signals.

\section{A STARING RADAR NETWORK}

In this section we describe the implementation of a small radar network that exploits a staring mode of operation for each transmit node and multiple fixed beams on receive.

Staring radars have attracted particular interest in recent years due to the advantage they offer for surveillance of small airborne targets such as drones [11, 12, 13, 17]. L-band staring systems as detailed in [14] use a broad beam antenna on transmit and an array of 2-D receivers that are digitised at element level to enable digital beam-forming on receive. The radar recorded data from each channel (64 in total) for each range bin and pulse is processed to form spatial beams. The time samples from each range and angular bin are coherently integrated to obtain Doppler data. The non-scanning configuration allows extended dwell on targets over the entire field of regard which provides the necessary sensitivity for reliable detection of drones, birds and other small objects in strong clutter [15]. The angular coverage is bounded by the antenna aperture and networking multiple staring radars enables both extension of the coverage area along with other key advantages such as improved sensitivity and resolution as discussed in the previous section.

The practical challenge with the networking a set of staring radars is to understand how to ensure full coherence between all the nodes in the network. Each radar is driven by a reference oscillator that is the source of the carrier frequency generation but also provides coherence between the transmitted waveform and the received echo. For multiple radars to operate coherently they must either be driven by the same reference oscillator or be able to operate with highly stable clocks that are able to maintain coherence between noncollocated nodes. Whilst the aim is to seek full coherence between all the nodes in the radar network, preliminary experiments were undertaken to evaluate the performance that can be achieved with a networked radar without using, in this example, any direct "wired" synchronisation.

The experimental set-up comprised two staring radars, separated by about $1 \mathrm{~km}$ as shown in Fig. . Both radars were tuned to the same L-Band carrier frequency. The primary radar was both transmitting and receiving. It was set to a pulse repetition interval of $136 \mu$ secs. The timing of each pulse is controlled by the clock counter provided by the reference oscillator. The received signal, once down-converted to baseband, is then matched filtered with a digital copy of the transmitted pulse. The match filter delay is set based on an integer number of clock-counts from the time of the transmitted pulse so that the first range gate corresponds to a distance equal to the start of the radar range swath. For the transmit radar, the delay corresponding to the location of the start of the range swath is set to $1.5 \mu$ secs. This way the range gates are correctly positioned in relation to the radar's true location. The primary radar captured data corresponding to range swath of $5 \mathrm{~km}$ which equates to $33 \mu$ secs duration. For the remaining $103 \mu$ secs of the pulse interval, the receiver remains silent and captures no data. The data capture is initiated again at the next pulse transmission and the cycle is repeated. For the fully coherent primary radar the 64 range gates are perfectly aligned with respect to the start range for each pulse. This represents the classic operation of a monostatic radar.

The second radar was also configured to the same carrier frequency as the primary radar. This radar also used the identical digital copy of the pulse to use in match filtering. Whilst the second radar operated at the same reference oscillator frequency, as there was no direct synchronisation there was no coherency between the two radar. For this second radar the range swath was set to $10 \mathrm{~km}$ so the data capture duration per pulse is $68 \mu \mathrm{secs}$. With no time alignment between the two radar sample clocks, the passive radar data capture sequence started essentially at random times compared to the transmitted pulse timing. The pulse repetition interval on the second radar was also set to $136 \mu$ secs, same as that for the primary radar. This was limited as a result of the maximum write speed of the data disk to which raw data was recorded. In summary, the configuration of the second radar is that it captured data for $68 \mu \mathrm{secs}$, remained silent for a further $68 \mu$ secs and then repeated the data capture. Within one pulse repetition interval of the second radar, a single pulse from the primary radar would have been transmitted. With a capture window of only half as long as the pulse repetition interval this equated to the passive radar only being able to align its capture window for about $50 \%$ of the time with the transmit pulse. Note, no reference channel is used to capture the direct path and the matched filter using a digital copy of the transmitted pulse is performed on the echo received by the passive receiver.

A key challenge is how to align the pulse delay that is exercised to initiate the match filtering on the second radar with the transmit pulse time of the primary radar. The clock count generated by the second radar will have not only a random offset with respect to the clock count of the primary radar but the two clock counts will drift over time and introduce further mis-alignment. Whilst a correction for the clock count offset and correction for drift will require full coherence between the network, preliminary results are presented without coherence to assess the signal quality that would be achieved with a non-coherent network.

Both radars were operating over several periods spanning two days and data was recorded for a number of flights with control targets. In addition to the control targets there were also a number of opportune aircrafts that flew within the field of view of the radar during the trial. The primary radar was processing the monostatic data in real-time and outputting tracks of the detected targets and several of such tracks were noted during the course of the trial. Fig. 8 shows section of a track from one of these opportune aircraft. The target had taken off from the runway at the airfield and the radar tracker reported the target to be flying outbound until eventually it went out of range. 
Raw data was recorded for both radars and processed offline to analyse the range-Doppler output obtained with each radar node. GPS data from the control targets were used to identify the target echoes in the raw data. Similarly, the position estimates from the tracker output were used to extract raw data for any target for which radar tracks were obtained. Information on the target position as a function of time is used to form a beam centred on the target for each range bin through digital beam forming. The beam formed data from 2048 pulses for all the range delay samples are processed using an FFT to generate one range-Doppler frame. The process is repeated over multiple frames and the range-Doppler data is analysed to evaluate the output from both radar receivers for the duration of the track.

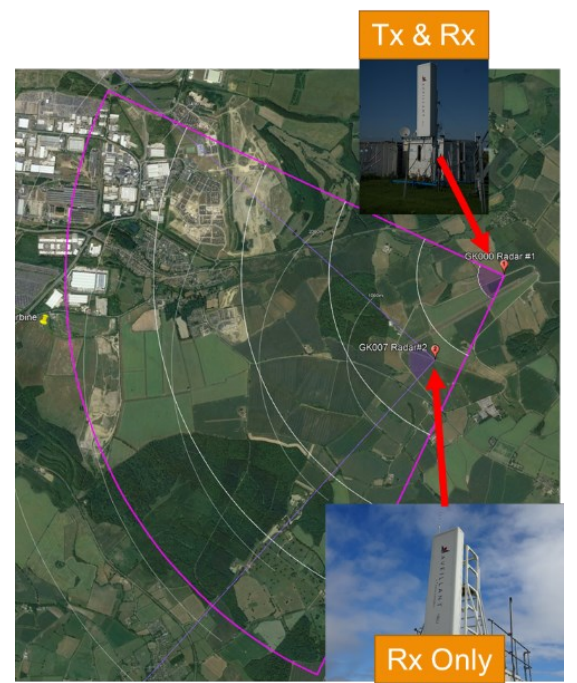

Fig. 7 Experimental set-up for two staring radar network

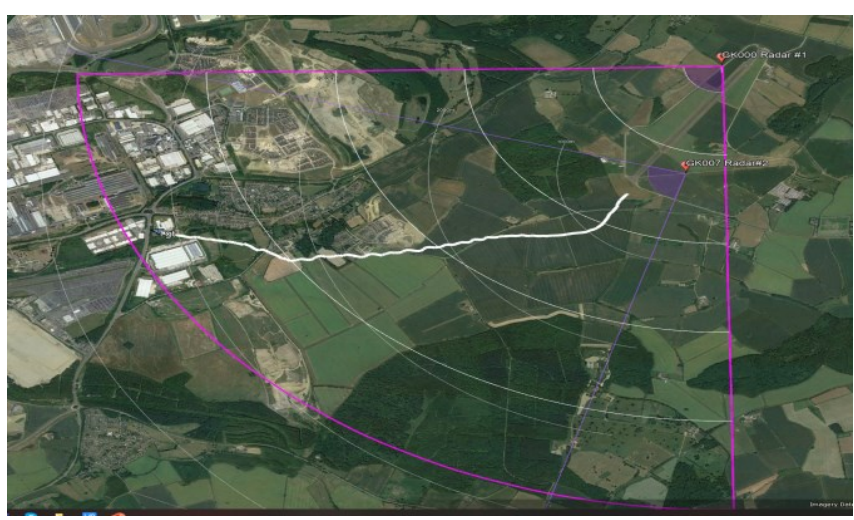

Fig. 8 Track of target of opportunity travelling out-bound

Firstly, the spectrogram of the target obtained with the primary radar is presented in Fig. 9. The spectrogram plots the Doppler for each frame at the range bin as reported by the tracker. In this plot the strong echo at zero Doppler is from stationary ground clutter and the dominant peak at $20-30 \mathrm{~m} / \mathrm{s}$ is from the target body Doppler. Note there are micro-Doppler sidebands which are characteristic of a rotary wing target and this is consistent with previous published results with this type of radar [16]. Next is shown a range-Doppler plot for the beam that is centred on the target for one frame (see Fig. 10). Radial velocity in $\mathrm{m} / \mathrm{s}$ is plotted along the $\mathrm{x}$-axis and the vertical axis is the range bin index where each range bin corresponds to roughly $75 \mathrm{~m}$ spacing. There is strong clutter present at near range. The chosen frame is near the start of the track where the target is closest to the radar. The target location obtained from the tracker is marked by the red cross. This marks the exact location of the target body Doppler. The measured SNR is close to $60 \mathrm{~dB}$. As the target heads further out in range its SNR drops and it can be observed that the sidebands are fading out as well. The variation in the measured SNR ranged from $60 \mathrm{~dB}$ to $30 \mathrm{~dB}$ over the length of the track. Overall, the results from the monostatic radar are well within the expected signal level anticipated from a manned aircraft at short ranges.

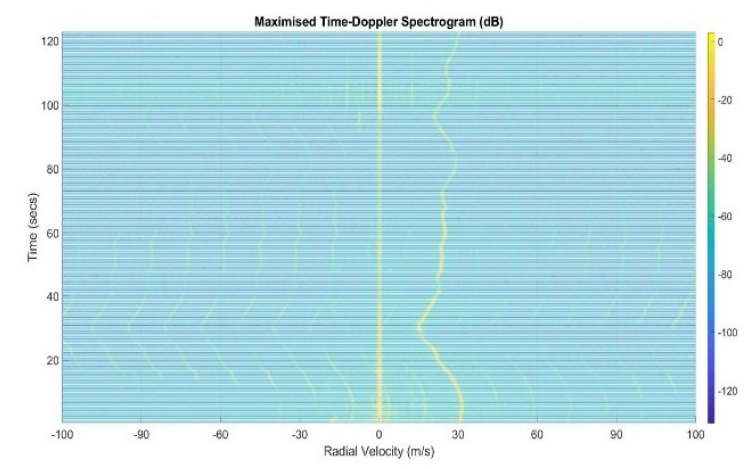

Fig. 9 Doppler spectrogram of an aircraft observed with the monostatic system

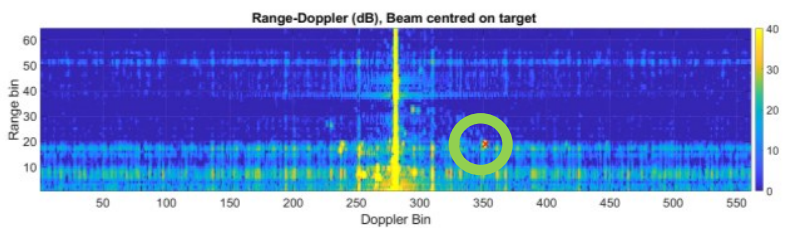

Fig. 10 Range Doppler output from primary radar showing the echo from target of opportunity (green circle \& red cross)

Next, the output obtained with the second radar that is only operating as a passive receive system is shown in Fig. 11. The range-Doppler plot is taken from approximately the same time instance as the data presented in Fig. 10. The position estimated from the monostatic radar track is used to select the estimated location of the target in the bi-static data. The bistatic Doppler will be near identical to the monostatic Doppler as in this instance the target is traveling along the radial path with respect to both radars. The bistatic range will be less than the monostatic range, however in this case there will be an additional misalignment between measured and actual bistatic range due to the sample clock offset with respect to the transmitted pulse. This is evident in the measured rangeDoppler plot of Fig. 11 where the initial half range bins have noise only data. The range bin at which the clutter starts can be used as a coarse alignment for the start of the range gate. It does mean that only part of the $10 \mathrm{~km}$ range swath captured by the passive radar will have valid data.

This is further evident in the spectrogram (Fig. 12) which is generated for the same time span as for the spectrogram in Fig. 9. In the bi-static spectrogram the target echo is lost halfway along the time span whilst the target remains present in the monostatic data recorded over the same period. Also evident is the broader Doppler spread in both the target and clutter Doppler compared with the monostatic spectrogram. This is due to the non-coherent nature of the current experimental set-up. However, despite the lack of coherence, the SNR is sufficiently high to even observe the microDoppler sidebands. A further observation made with this data set is that the SNR fluctuations differs between the bistatic and 
monostatic measurements. Evidently, this is one of the motivations for network systems to exploit the difference in SNR to overcome signal fading.

The results show that even a passive receiver, albeit with crude time alignment, allows sufficient processing gain to detect echoes of a target illuminated by the transmitter component of the network. Further work is required to understand what additional processing enhancements will enable aspects such as removal of correction for the offset error in range within the network geometry. Modelling will aid understanding of the predicted range-Doppler response that is expected for the given radar geometries. Future work will explore the level of synchronisation tolerance that will be required to achieve optimal processing gain and maximise range, angle and Doppler accuracy.

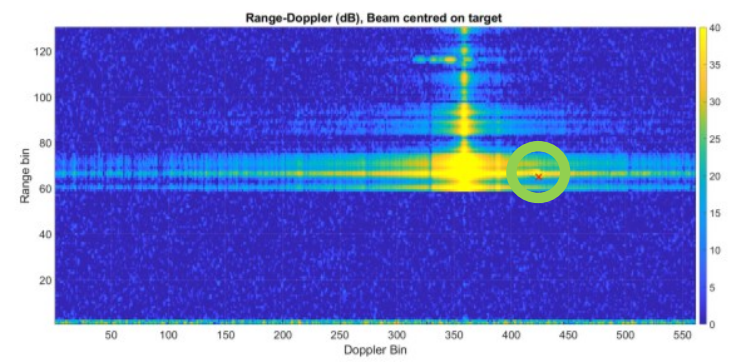

Fig. 11 Range Doppler output from the passive bistatic radar showing the echo from the target of opportunity (green circle \& red cross)

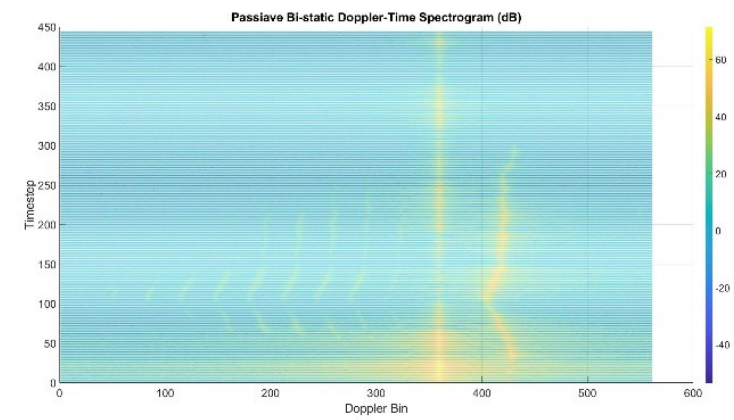

Fig. 12 Doppler spectrogram of an aircraft observed with the passive system

\section{CONCLUSION}

In this paper we report initial aspects of the performance of networked staring radar that are related to the location and optimum processing of signals under the assumption of perfect coherency. We also report early experiments exploiting a pair of staring radars, that form the fundamental bistatic unit of a networked radar, in order to explore options for levels of distributed coherency as a function of method used to establish that coherency. Here we show that a passive mode of non-coherent operation is sufficiently successful to enable target detection in both receive nodes of the network, but further research is required to evaluate the degree of achieved coherency when the system is fully synchronised. This will provide a benchmark against which other methods can be compared and ultimately how these can be combined with cognitive processing to enhance system performance.

\section{ACKNOWLEDGMENTS}

The work was part funded by DSTL and supported by an Aveillant Limited funded Industrial $\mathrm{PhD}$ with Cranfield University. The authors would also like to thank Aveillant Limited for supporting the radar trials.

\section{REFERENCES}

[1] S. Ansari, S. Del Greco, E. Kearns, O. Brown, S. Wilkins, M. Ramamurthy, J. Weber, R. May, J. Sundwall, J. Layton, A. Gold, A. Pasch and V. Lakshmanan, "Unlocking the potential of NEXRAD data through NOAA's big data partnership", Bull. Am. Metrorol. Soc., 2018, vol 99, pp. 189-204.

[2] A. Huuskonen, E. Saltikoff and I. Holleman, "The operational weather radar network in Europe”, Bull. Am. Metrorol. Soc., 2014, vol 95, pp. 897-907.

[3] J. Shmoun-Baranes, C. Nilsson, S. Bauer and J. Chapman, "Taking radar aeroecology into the $21^{\text {st }}$ centuary", Ecography, 2019, vol. 42, pp. 847-851.

[4] H. Roarty et al, "The global high frequency radar network", Front. Mar. Sci., May 2019, https://doi.org/10.3389/fmars.2019.00164

[5] S. Hamed Javadi and A. Farina, "Radar networks: A review of features and challenges", Information fusion, vol. 61, 2020, pp. 48-55.

[6] T.E. Derham, S. Doughty, K. Woodbridge and C.J. Baker, "Design and evaluation of a low-cost multistatic radar system", IET, Radar, Sonar and Navigation, 2007, vol. 1(5), pp. 362-368.

[7] W. A. Al-Ashwal, C. J. Baker, A. Balleri, H. D. Griffiths, R. Harmanny, M. Inggs, W. J. Miceli, M. Ritchie, J. S. Sandebergh, A. Stove, R. J. A. Tough, K. D. Ward, S. Watts and K. Woodbridge." Statistical analysis of simultaneous monostatic and bistatic sea clutter at low grazing angles", IET Electronics letters, 2011, 47(10), pp. 621622.

[8] G.E. Smith, Z. Cammenga, A Mitchell, K.A. Bell, J. Johnson, M. Rangaswamy, C.J. Baker, "Experiments with cognitive radar", IEEE Aerospace and Elctronic System Magazine, 2016, vol 31(12), pp. 3446.

[9] H L Van Trees. Detection, estimation, and modulation theory, Part 3 Radar-sonar signal processing and Gaussian signals in noise, volume 18. John Wiley, 1972.

[10] Steven M Kay 1951-. Fundamentals of statistical signal processing: estimation theory / Steven M. Kay. PTR Prentice Hall, c1993., 199

[11] M. Jahangir, C. J. Baker and G. K. Oswald, "Doppler characteristics of micro-drones with L-band multibeam staring radar", IEEE RadarCon 2017, Seattle, US, May 2017

[12] M. Jahangir M and C. J. Baker, "Results from U-space live test flight trials evaluating non-cooperative surveillance of drones using an Lband staring radar", International Radar Symposium IRS 2019, Ulm, Germany, Jun. 2019

[13] M. Jahangir, B. I. Ahmad B I and C. J. Baker, "Robust Drone Classification Using Two-Stage Decision Trees and Results from SESAR SAFIR Trials", 2020 IEEE International Radar Conference, Washington DC, US, Apr 2020

[14] M. Jahangir, "Target centric wide-area 3-D surveillance using a nonscanning multibeam receiver array", Proc. IEEE International Radar Conference, Arlington, US, 11-15 May 2015

[15] M. Jahangir and C. J. Baker, "L-band staring radar performance against micro-drones", International Radar Symposium IRS 2018, Bonn, Germany, Jun. 2018

[16] C. Bennett, M. Jahangir, F. Fioranelli, B. I. Ahmad and J. L. Kernec, "Use of Symmetrical Peak Extraction in Drone Micro-Doppler Classification for Staring Radar", 2020 IEEE Radar Conference, Florence, Italy, Sep 2020

[17] S. A. Harman, "A comparison of staring radars with scanning radars for UAV detection: Introducing the Alarm staring radar", In the proceedings of the European radar conference (EuRAD), pp. 185-188, 2015

[18] B. Griffin, A. Balleri, C. J. Baker, and M. Jahangir, "Optimal receiver placement in staring cooperative radar networks for detection of drones", 2020 IEEE Radar Conference, Florence, Italy, Sep 2020 